\title{
Quasi-uniform structures determined by closure operators ${ }^{\text {th }}$
}

\author{
David Holgate, Minani Iragi *,1 \\ Department of Mathematics and Applied Mathematics, University of the Western Cape, Bellville 7535, \\ South Africa
}

\section{A R T I C L E I N F O}

Article history:

Received 17 September 2019

Received in revised form 9 February

2021

Accepted 16 March 2021

Available online 19 March 2021

\section{$M S C$ :}

18A20

$54 \mathrm{~A} 15$

$54 \mathrm{~B} 30$

$54 \mathrm{E} 15$

Keywords:

Closure operator

Syntopogenous structure

Quasi-uniform structure

\begin{abstract}
A B S T R A C T
We demonstrate a one-to-one correspondence between idempotent closure operators and the so-called saturated quasi-uniform structures on a category $\mathcal{C}$. Not only this result allows to obtain a categorical counterpart $\mathbb{P}$ of the Császár-Pervin quasiuniformity $\mathcal{P}$, that we characterize as a transitive quasi-uniformity compatible with an idempotent interior operator, but also permits to describe those topogenous orders that are induced by a transitive quasi-uniformity on $\mathcal{C}$. The categorical counterpart $\mathbb{P}^{*}$ of $\mathcal{P}^{-1}$ is characterized as a transitive quasi-uniformity compatible with an idempotent closure operator. When applied to other categories outside topology $\mathcal{P}$ allows, among other things, to generate a family of idempotent closure operators on Grp, the category of groups and group homomorphisms, determined by the normal closure.
\end{abstract}

() 2021 Elsevier B.V. All rights reserved.

\section{Introduction}

The Császár-Pervin ([13,2]) fundamental result that every topological space admits at least one quasiuniformity motivated the study of quasi-uniform structures compatible with a given topology (see e.g. [7] with references therein). It is this great generality of quasi-uniformities which allows one to view in some sense the study of quasi-uniform spaces as an alternative approach to the study of topological spaces. In $[12,8]$ a quasi-uniform structure is introduced on a category $\mathcal{C}$ as a suitable endofunctor category on the subobject lattice, subX, for each object $X$ in $\mathcal{C}$ and characterized as an appropriate family of closure operators ([5]). It is therefore not hard to observe that every quasi-uniformity on $\mathcal{C}$ induces a closure operator

\footnotetext{
This work was supported by the South African National Research Foundation.

* Corresponding author.

E-mail addresses: dholgate@uwc.ac.za (D. Holgate), 3418181@myuwc.ac.za (M. Iragi).

1 The final corrections of the paper were done when the corresponding author was holding a lecturer position at University of Lubumbashi.
} 
(resp. interior [14]) in a natural way. This motivates us to ask if given a closure operator (resp. interior) on a category one can always find at least a quasi-uniformity which generates that closure operator (resp. interior). The present paper aims to address this question. We show that there is a subconglomerate of the conglomerate of all quasi-uniform structures on $\mathcal{C}$ which is order isomorphic to the conglomerate of all idempotent closure operators. With the help of categorical topogenous structures ([9]), we demonstrate that for any idempotent interior operator $i$ (closure $c$ ) on $\mathcal{C}$, there is at least a transitive quasi-uniformity which generates $i(c)$. A condition under which a topogenous order is compatible with a transitive quasiuniformity is found. This allows us to characterize closure operators (resp. interior) that are compatible with a transitive quasi-uniformity.

\section{Preliminaries}

Our primary reference for categorical terminology is [1]. For the basic facts on categorical closure and interior operators we refer to [4,5] and [14]. Concerning the categorical topogenous, quasi-uniform and syntopogenous structures, we use $[12,8,9]$. Throughout the paper, we consider a category $\mathcal{C}$ supplied with a proper $(\mathcal{E}, \mathcal{M})$-factorization system for morphisms. The category $\mathcal{C}$ is assumed to be $\mathcal{M}$-complete so that pullbacks of $\mathcal{M}$-morphisms along $\mathcal{C}$-morphisms and arbitrary $\mathcal{M}$-intersections of $\mathcal{C}$-morphisms exist and are again in $\mathcal{M}$. For any $X \in \mathcal{C}, \operatorname{sub} X=\{m \in \mathcal{M} \mid \operatorname{cod}(m)=X\}$. It is ordered as follows: $n \leq m \Leftrightarrow$ $\exists j \mid m \circ j=n$. If $m \leq n$ and $n \leq m$ then they are isomorphic. We shall simply write $m=n$ in this case. $\operatorname{Sub} X$ is a (possibly large) complete lattice with greatest element $1_{X}: X \longrightarrow X$ and the least element $0_{X}: O_{X} \longrightarrow X$. Any $\mathcal{C}$-morphism, $f: X \longrightarrow Y$ induces an image/pre-image adjunction

$$
f(m) \leq n \Leftrightarrow m \leq f^{-1}(n)
$$

for all $n \in \operatorname{sub} Y, m \in \operatorname{sub} X$ with $f(m)$ the $\mathcal{M}$-component of the $(\mathcal{E}, \mathcal{M})$-factorization of $f \circ m$ while $f^{-1}(n)$ is the pullback of $n$ along $f$. We have from the image/pre-image adjunction that $f\left(f^{-1}(n)\right) \leq n$ (with $f\left(f^{-1}(n)\right)=n$ if $f \in \mathcal{E}$ and $\mathcal{E}$ is pullback stable along $\mathcal{M}$-morphisms) and $m \leq f^{-1}(f(m)$ ) (with $m=f^{-1}(f(m))$ if $\left.f \in \mathcal{M}\right)$ for any $n \in \operatorname{sub} Y$ and $m \in \operatorname{sub} X$. We shall sometimes find it important to assume that for any $\mathcal{C}$-morphism $f: X \longrightarrow Y$, the inverse image $f^{-1}$ commutes with the joins of subobjects so that it has a right adjoint $f_{*}$ given by $f_{*}(m)=\bigvee\left\{n \in \operatorname{sub} Y \mid f^{-1}(n) \leq m\right\}$. Thus $f^{-1}(n) \leq m \Leftrightarrow n \leq$ $f_{*}(m), f^{-1}\left(f_{*}(m)\right) \leq m\left(\right.$ with $f^{-1}\left(f_{*}(m)\right)=m$ if $\left.f \in \mathcal{M}\right)$ and $n \leq f_{*}\left(f^{-1}(n)\right)$ (with $f_{*}\left(f^{-1}(n)\right)=n$ if $f \in$ $\mathcal{E}$ and $\mathcal{E}$ stable under pullbacks).

Definition 2.1. A topogenous order $\sqsubset$ on $\mathcal{C}$ is a family $\left\{\sqsubset_{X} \mid X \in \mathcal{C}\right\}$ of relations, each $\sqsubset_{X}$ on subX, such that:

(T1) $m \sqsubset_{X} n \Rightarrow m \leq n$ for every $m, n \in \operatorname{sub} X$,

(T2) $m \leq n \sqsubset_{X} p \leq q \Rightarrow m \sqsubset_{X} q$ for every $m, n, p, q \in \operatorname{sub} X$, and

(T3) every morphism $f: X \longrightarrow Y$ in $\mathcal{C}$ is $\sqsubset$-continuous, $m \sqsubset_{Y} n \Rightarrow f^{-1}(m) \sqsubset_{X} f^{-1}(n)$ for every $m, n \in \operatorname{sub} Y$.

Given two topogenous orders $\sqsubset$ and $\sqsubset^{\prime}$ on $\mathcal{C}$, $\sqsubset \subseteq \sqsubset^{\prime}$ if and only if $m \sqsubset{ }_{X} n \Rightarrow m \sqsubset_{X}^{\prime} n$ for all $m, n \in \operatorname{sub} X$ The resulting ordered conglomerate of all topogenous orders on $\mathcal{C}$ is denoted by $\operatorname{TORD}(\mathcal{C}, \mathcal{M})$.

A topogenous order $\sqsubset$ is said to be

(1) $\bigwedge$-preserving if $\left(\forall i \in I: m \sqsubset_{X} n_{i}\right) \Rightarrow m \sqsubset_{X} \bigwedge n_{i}$;

(2) $\bigvee$-preserving if $\left(\forall i \in I: m_{i} \sqsubset_{X} n\right) \Rightarrow \bigvee m_{i} \sqsubset_{X} n$;

(3) interpolative if $m \sqsubset_{X} n \Rightarrow \exists p \mid m \sqsubset_{X} p \sqsubset_{X} n$ for all $X \in \mathcal{C}$. 
The ordered conglomerate of all $\Lambda$-preserving and $\bigvee$-preserving topogenous orders is denoted by $\bigwedge-\operatorname{TORD}(\mathcal{C}, \mathcal{M})$ and $\bigvee-T O R D(\mathcal{C}, \mathcal{M})$ respectively. $\bigwedge-\operatorname{INTORD}(\mathcal{C}, \mathcal{M})($ resp. $\bigwedge-\operatorname{INTORD}(\mathcal{C}, \mathcal{M}))$ will denote the conglomerate of all interpolative meet preserving (resp. join preserving) topogenous orders.

Definition 2.2. A closure operator $c$ on $\mathcal{C}$ with respect to $\mathcal{M}$ is given by a family of maps $\left\{c_{X}: \operatorname{sub} X \longrightarrow\right.$ $\operatorname{sub} X \mid X \in \mathcal{C}\}$ such that:

(C1) $m \leq c_{X}(m)$ for all $m \in \operatorname{sub} X$;

(C2) $m \leq n \Rightarrow c_{X}(m) \leq c_{X}(n)$ for all $m, n \in \operatorname{sub} X$;

(C3) every morphism $f: X \longrightarrow Y$ is $c$-continuous: $f\left(c_{X}(m)\right) \leq c_{Y}(f(m))$ for all $m \in \operatorname{sub} X$.

We denote by $C L(\mathcal{C}, \mathcal{M})$ the conglomerate of all closure operators on $\mathcal{C}$ with respect to $\mathcal{M}$ ordered as follows: $c \leq c^{\prime}$ if $c_{X}(m) \leq c_{X}^{\prime}(m)$ for all $m \in \operatorname{sub} X$ and $X \in \mathcal{C}$. A closure operator $c$ on $\mathcal{C}$ is idempotent if $c_{X}\left(c_{X}(m)\right)=c_{X}(m)$ for all $m \in \operatorname{sub} X$ and $X \in \mathcal{C} . I C L(\mathcal{C}, \mathcal{M})$ will denote the conglomerate of all idempotent closure operators on $\mathcal{C}$. For any $c \in C L(\mathcal{C}, \mathcal{M})$, there is a least idempotent closure operator $\hat{c} \geq c$, the idempotent hull of $c$. A subobject of an object $X \in \mathcal{C}$ is said to be $c$-closed if $c_{X}(m)=m$.

Definition 2.3. An interior operator $i$ on $\mathcal{C}$ with respect to $\mathcal{M}$ is given by a family of maps $\left\{i_{X}: \operatorname{sub} X \longrightarrow\right.$ $\operatorname{sub} X \mid X \in \mathcal{C}\}$ such that:

(I1) $i_{X}(m) \leq m$ for all $m \in \operatorname{sub} X$ and $X \in \mathcal{C}$;

(I2) $m \leq n \Rightarrow i_{X}(m) \leq i_{X}(n)$ for all $m, n \in \operatorname{sub} X, X \in \mathcal{C}$;

(I3) every morphism $f: X \longrightarrow Y$ is $i$-continuous: $f^{-1}\left(i_{Y}(n)\right) \leq i_{X}\left(f^{-1}(n)\right)$ for all $n \in \operatorname{subY}$.

$\operatorname{INT}(\mathcal{C}, \mathcal{M})$ will denote the conglomerate of all interior operators on $\mathcal{C}$ with respect to $\mathcal{M}$. It is ordered as follows: $i \leq i^{\prime}$ if $i_{X}(m) \leq i_{X}^{\prime}(m)$ for all $m \in \operatorname{sub} X, X \in \mathcal{C}$. A subobject of an object $X \in \mathcal{C}$ is said to be $i$-open if $i_{X}(m)=m$. An interior operator $i$ is said to be idempotent provided that $i_{X}(m)$ is $i$-open for every $m \in \operatorname{sub} X$ and $X \in \mathcal{C}$. We write $\operatorname{IINT}(\mathcal{C}, \mathcal{M})$ the ordered conglomerate of all idempotent interior operators on $\mathcal{C}$.

Associated closure and interior operators provide an equivalent description a topology. This natural correspondence between these two notions no longer holds in abstract categorical settings since the subobject lattices are not necessarily boolean algebras. The categorical topogenous order that is defined above allows to obtain a nice relationship between closure and interior operators on a category in the sense that many concepts and definitions that have been studied separately for categorical closure and interior operators can be shown to be exactly the same using topogenous orders (see for example the notion of $\sqsubset$-strict subobject defined and used in third section of this paper). The next result that we recall from ([9]) exhibits the clear relationship between closure, interior and topogenous order in a category while it is also known from the same paper that a topogenous order on $\mathcal{C}$ is basically equivalent to a neighbourhood operator ([10]).

\section{Proposition 2.1.}

(1) $\bigvee-T O R D(\mathcal{C}, \mathcal{M})$ is order isomorphic to $\operatorname{INT}(\mathcal{C}, \mathcal{M})$. The inverse assignments of each other are given by $i_{X}(m)=\bigvee\left\{p \mid p \sqsubset_{X} m\right\}$ and $m \sqsubset_{X}^{i} n \Leftrightarrow m \leq i_{X}(n)$ for all $X \in \mathcal{C}$.

(2) $\bigwedge-\operatorname{TORD}(\mathcal{C}, \mathcal{M})$ is order isomorphic to $C L(\mathcal{C}, \mathcal{M})$. The inverse assignments of each other are given by $c_{X}^{\sqsubset}(m)=\bigwedge\left\{p \mid m \sqsubset_{X} p\right\}$ and $m \sqsubset_{X}^{c} n \Leftrightarrow c_{X}(m) \leq n$ for all $X \in \mathcal{C}$.

It is well known (see e.g. [6]) that an (entourage) quasi-uniformity on $\mathrm{X}$ can be equivalently expressed as an appropriate family of maps $U: X \longrightarrow \mathcal{P}(X)$. Since these maps can easily be extended to endomaps on 
$\mathcal{P}(X)$, it is possible to think of a quasi-uniformity on $\mathcal{C}$ as a suitable family of endomaps on $\operatorname{sub} X$ for each $X \in \mathcal{C}$. This is the point expressed in Definition 2.4. Let us denote by $\mathcal{F}(\operatorname{sub} X)$ the endofunctor category on $\operatorname{sub} X$ for each $X \in \mathcal{C}$. It is clear that for all $U, V \in \mathcal{F}(\operatorname{sub} X), U \leq V$ if $U(m) \leq V(m)$ for all $m \in \operatorname{sub} X$.

Definition 2.4. A quasi-uniformity on $\mathcal{C}$ with respect to $\mathcal{M}$ is a family $\mathcal{U}=\left\{\mathcal{U}_{X} \mid X \in \mathcal{C}\right\}$ with $\mathcal{U}_{X}$ a full subcategory of $\mathcal{F}(\operatorname{sub} X)$ for each $X$ such that:

(U1) For any $U \in \mathcal{U}_{X}, 1_{X} \leq U$.

(U2) For any $U \in \mathcal{U}_{X}$, there is $U^{\prime} \in \mathcal{U}_{X}$ such that $U^{\prime} \circ U^{\prime} \leq U$.

$(U 3)$ For any $U \in \mathcal{U}_{X}$ and $U \leq U^{\prime}, U^{\prime} \in \mathcal{U}_{X}$.

(U4) For any $U, U^{\prime} \in \mathcal{U}_{X}, U \wedge U^{\prime} \in \mathcal{U}_{X}$.

$(U 5)$ For any $\mathcal{C}$-morphism $f: X \longrightarrow Y$ and $U \in \mathcal{U}_{Y}$, there is $U^{\prime} \in \mathcal{U}_{X}$ such that $f\left(U^{\prime}(m)\right) \leq U(f(m))$ for all $m \in \operatorname{sub} X$.

We shall denote by $Q U N I F(\mathcal{C}, \mathcal{M})$ the conglomerate of all quasi-uniform structures on $\mathcal{C}$. It is ordered as follows: $\mathcal{U} \leq \mathcal{V}$ if for all $X \in \mathcal{C}$ and $U \in \mathcal{U}_{X}$, there is $V \in \mathcal{V}_{X}$ such that $V \leq U$. In most cases we describe a quasi-uniformity by defining a base for it. A base for a quasi-uniformity $\mathcal{U}$ on $\mathcal{C}$ is a family $\mathcal{B}=\left\{\mathcal{B}_{X} \mid X \in \mathcal{C}\right\}$ with each $\mathcal{B}_{X}$ a full subcategory of $\mathcal{F}(\operatorname{sub} X)$ for all $X \in \mathcal{C}$ satisfying all the axioms in Definition 2.4 except $(U 3)$. If $\mathcal{B}_{X}$ for any $X \in \mathcal{C}$ is a base element with a single member $V$, we shall write $V_{X}$. A base for quasiuniformity on $\mathcal{C}$ is transitive if for all $X \in \mathcal{C}$ and $U \in \mathcal{B}_{X}, U \circ U=U$. A quasi-uniformity with a transitive base is called a transitive quasi-uniformity. The ordered conglomerate of all transitive quasi-uniformities on $\mathcal{C}$ will be denoted by $T Q U N I F(\mathcal{C}, \mathcal{M})$. A quasi-uniformity $\mathcal{U}$ on $\mathcal{C}$ is a uniformity provided that for every $U \in \mathcal{U}_{X}$ and $X \in \mathcal{C}$, there is $V \in \mathcal{U}$ such that $m \leq U(n) \Leftrightarrow n \leq V(m)$ for any $m, n \in$ sub $X$. We denote by $\operatorname{UNIF}(\mathcal{C}, \mathcal{M})$ the conglomerate of all uniform structures on $\mathcal{C}$. It is also clear that if $U \in \mathcal{U}_{X}$ for any $X \in \mathcal{C}$ then $U(m) \geq 0_{X}$ for all $m \in \operatorname{sub} X$, otherwise any $U \in \mathcal{F}(\operatorname{sub} X)$ can belong to $\mathcal{U}_{X}$ since $0_{X}$ is the least element of $\operatorname{sub} X$. The unique $U \in \mathcal{F}(\operatorname{sub} X)$ such that $U(m)=1_{X}$ for any $m \in \operatorname{sub} X$ will be denoted by $\mathbf{1}_{\mathbf{X}}$ while $\mathbf{d}_{\mathbf{X}}$ will denote the map $U \in \mathcal{F}(\operatorname{sub} X)$ with the property that $U(m)=m$.

Definition 2.5. A syntopogenous structure on $\mathcal{C}$ with respect to $\mathcal{M}$ is a family $\mathcal{S}=\left\{\mathcal{S}_{X} \mid X \in \mathcal{C}\right\}$ such that each $\mathcal{S}_{X}$ a family of relations on $\operatorname{sub} X$ satisfying:

$(S 1) \sqsubset_{X}$ is a relation on $\operatorname{subX}$ satisfying $(T 1)$ and $(T 2)$.

$(S 2) \mathcal{S}_{X}$ is a directed set with respect to inclusion.

$(S 3) \sqsubset_{X}=\bigcup \mathcal{S}_{X}$ is an interpolative topogenous order.

The ordering of topogenous orders can be extended to syntopogenous structures in the following way: $\mathcal{S} \leq \mathcal{S}^{\prime}$ if for all $X \in \mathcal{C}$ and $\sqsubset{ }_{X} \in \mathcal{S}_{X}$, there is $\sqsubset_{X}^{\prime} \in \mathcal{S}^{\prime}{ }_{X}$ such that $\sqsubset_{X} \subseteq^{\prime} \sqsubset_{X}^{\prime}$. The resulting conglomerate will be denoted by $S Y N T(\mathcal{C}, \mathcal{M}) . \mathcal{S} \in S Y N T(\mathcal{C}, \mathcal{M})$ is co-perfect if each $\sqsubset_{X} \in \mathcal{S}_{X}$ is $\bigwedge$-preserving for all $X \in \mathcal{C}$. It is interpolative if every $\sqsubset_{X} \in \mathcal{S}_{X}$ interpolates. The ordered conglomerate of all interpolative co-perfect syntopogenous structures will be denoted by $\operatorname{INTCSYNT}(\mathcal{C}, \mathcal{M})$.

Theorem 2.2. TQUNIF $(\mathcal{C}, \mathcal{M})$ is order isomorphic to $\operatorname{INTCSYNT}(\mathcal{C}, \mathcal{M})$. The inverse morphisms of each other $\mathcal{U} \longmapsto \mathcal{S}^{\mathcal{U}}$ and $\mathcal{S} \longmapsto \mathcal{U}^{\mathcal{S}}$ are given by

$$
\begin{aligned}
& \mathcal{S}_{X}^{\mathcal{B}}=\left\{\sqsubset_{X}^{U} \mid U \in \mathcal{B}_{X}\right\} \text { where } m \sqsubset_{X}^{U} n \Leftrightarrow U(m) \leq n, \text { and } \\
& \mathcal{B}_{X}^{\mathcal{S}}=\left\{U^{\sqsubset} \mid \sqsubset_{X} \in \mathcal{S}_{X}\right\} \text { where } U^{\sqsubset}(m)=\bigwedge\left\{n \mid m \sqsubset_{X} n\right\}
\end{aligned}
$$

for all $X \in \mathcal{C}$ and $m, n \in \operatorname{subX}$. 
Since $\mathcal{S}_{X} \subseteq \wedge-T O R D(\mathcal{C}, \mathcal{M})$ for each $\mathcal{S} \in S Y N T(\mathcal{C}, \mathcal{M})$, it follows from Theorem 2.2 that a quasiuniformity on $\mathcal{C}$ is a collection of families of closure operators. By Proposition 2.1, $\bigwedge-\operatorname{INTORD}(\mathcal{C}, \mathcal{M})$ is isomorphic to the conglomerate of idempotent closure operators and from Theorem 2.2, $C S Y N T(\mathcal{C}, \mathcal{M}) \cong$ $Q U N I F(\mathcal{C}, \mathcal{M})$. Thus every idempotent closure operator on $\mathcal{C}$ is a base for a quasi-uniformity.

\section{Idempotent closure operators or saturated quasi-uniform structures}

Lemma 3.1. Let $\mathcal{U} \in Q U N I F(\mathcal{C}, \mathcal{M})$. Then $\mathcal{B}=\left\{\mathcal{B}_{X} \mid X \in \mathcal{C}\right\}$ is a base for $\mathcal{U}$ if and only if for any $U \in \mathcal{U}_{X}$, there is $V \in \mathcal{B}_{X}$ such that $V \leq U$.

A quasi-uniform structure $\mathcal{U}$ on $\mathcal{C}$ is said to be saturated if for any $X \in \mathcal{C}, \bigwedge\left\{U: U \in \mathcal{U}_{X}\right\} \in \mathcal{U}_{X}$. $\operatorname{SQUNIF}(\mathcal{C}, \mathcal{M})$ will denote the conglomerate of all saturated quasi-uniform structures on $\mathcal{C}$.

Proposition 3.2. Let $\mathcal{U} \in Q U N I F(\mathcal{C}, \mathcal{M})$ and $X \in \mathcal{C}$. Then $\mathcal{U} \in \operatorname{SQUNIF}(\mathcal{C}, \mathcal{M})$ if and only if there is a unique base $\mathcal{B}$ for $\mathcal{U}$ such that $\mathcal{B}_{X}$ has a single member.

Proof. Sufficiency is clear. Let $V(m)=\bigwedge\left\{U(m): U \in \mathcal{U}_{X}\right\}$ for any $m \in \operatorname{sub} X$. By assumption, $V \in \mathcal{U}_{X}$. Now, let $\mathcal{B}_{X}=\{V\}$. We must show that $V \circ V \leq V$ and satisfies (U5). Since $V \in \mathcal{U}_{X}$, there is $U \in \mathcal{U}_{X}$ such that $U \circ U \leq V$. But $V \leq U$ and so $V(V(m)) \leq V(U(m)) \leq U(U(m)) \leq V(m)$. Let $f: X \longrightarrow Y$ and $V_{Y} \in \mathcal{B}_{Y}$. Then $V_{Y} \in \mathcal{U}_{Y}$ and there is $U^{\prime}$ such that $f\left(U^{\prime}(m)\right) \leq V(f(m))$ for all $m \in \operatorname{sub} X$. Since $V_{X} \leq U^{\prime}$, $f\left(V_{X}(m)\right) \leq f\left(U_{X}^{\prime}(m)\right) \leq V(f(m))$. The uniqueness of $\mathcal{B}$ is easily seen.

As mentioned earlier, every idempotent closure operator is a base for a quasi-uniform structure on $\mathcal{B}$. Proposition 3.2, allows now to identify those quasi-uniform structures that are in one to one correspondence with idempotent closure operators.

Theorem 3.3. $\operatorname{SQUNIF}(\mathcal{C}, \mathcal{M})$ is order isomorphic to $\operatorname{ICL}(\mathcal{C}, \mathcal{M})$. The inverse assignments of each other $\mathcal{U} \longrightarrow c^{\mathcal{U}}$ and $c \longrightarrow \mathcal{U}^{c}$ are given by

$$
\begin{gathered}
\mathcal{U}_{X}^{c}=\left\{U \in \mathcal{F}(s u b X): c_{X} \leq U\right\} \text { and } \\
c_{X}^{\mathcal{U}}=\bigwedge\left\{U: U \in \mathcal{U}_{X}\right\}
\end{gathered}
$$

for all $X \in \mathcal{C}$

Proof. The assignment $c \longrightarrow \mathcal{U}^{c}$ is clearly well defined. For $\mathcal{U} \longrightarrow c^{\mathcal{U}}$, we only need to show that for any $U \in \operatorname{SQUNIF}(\mathcal{C}, \mathcal{M}), c^{\mathcal{U}} \circ c^{\mathcal{U}}=c^{\mathcal{U}}$. So for all $U \in \mathcal{U}_{X}$, there is $V \in \mathcal{U}_{X}$ such that $V \circ V \leq U$. Now, $V\left(c_{X}^{\mathcal{U}}(m)\right) \leq V(V(m)) \leq U(m)$. Thus $c_{X}^{\mathcal{U}}\left(c_{X}^{\mathcal{U}}(m)\right)=\bigwedge\left\{V\left(c_{X}^{\mathcal{U}}(m)\right): V \in \mathcal{U}_{X}\right\} \leq \bigwedge\left\{U(m): U \in \mathcal{U}_{X}\right\}=$ $c_{X}^{\mathcal{U}}(m)$. Let $c \in C L(\mathcal{C}, \mathcal{M}), c^{\mathcal{U}^{c}}(m)=\bigwedge\left\{U(m): U \in \mathcal{U}_{X}^{c}\right\}=c_{X}^{\mathcal{U}}(m)$ and for any $\mathcal{U} \in \operatorname{SQUNIF}(\mathcal{C}, \mathcal{M})$, $\mathcal{U}^{c^{\mathcal{U}}}=\left\{U \in \mathcal{F}(\operatorname{subX}): c_{X}^{\mathcal{U}} \leq U\right\}=\mathcal{U}$.

Recall that a morphism $f: X \longrightarrow Y$ is said to be $\mathcal{U}$-initial if for any $U \in \mathcal{U}_{X}$ there is $V \in \mathcal{U}_{Y}$ such that $f^{-1}(V(f(m))) \leq U(m)$ for all $m \in \operatorname{sub} X$. It is $c$-closed if $f\left(c_{X}(m)\right)=c_{Y}(f(m))$

Proposition 3.4. Let $\mathcal{U} \in \operatorname{SQUNIF}(\mathcal{C}, \mathcal{M})$ and $f: X \longrightarrow Y$ be a $\mathcal{C}$-morphism. Then

(1) $f$ is $\mathcal{U}$-initial if and only if $f^{-1}\left(c_{Y}^{\mathcal{U}}(f(m)) \leq c_{X}^{\mathcal{U}}(m)\right.$ for any $m \in \operatorname{sub} X$.

(2) $f$ is $c^{\mathcal{U}}$-closed if and only if for any $U \in \mathcal{U}_{X}$ there is $V \in \mathcal{U}_{Y}$ such that $\left.V(f(m)) \leq f(U(m))\right)$ for all $m \in \operatorname{sub} X$ 
Proof. (1) Assume $f$ is $\mathcal{U}$-initial. Then for any $U \in \mathcal{U}_{X}$ there is $V \in \mathcal{U}_{Y}$ such that $f^{-1}(V(f(m))) \leq U(m)$. Thus $f^{-1}\left(\bigwedge\left\{V(f(m)): V \in \mathcal{U}_{Y}\right\}\right) \leq U(m) \Rightarrow f^{-1}\left(c_{Y}^{\mathcal{U}}(f(m)) \leq \bigwedge\left\{U(m): U \in \mathcal{U}_{X}\right\}=c_{X}^{\mathcal{U}}(m)\right.$. Conversely if $f^{-1}\left(c_{Y}^{\mathcal{U}}(f(m)) \leq c_{X}^{\mathcal{U}}(m)\right.$ and $U \in \mathcal{U}_{X}$ then $f^{-1}\left(\bigwedge\left\{V(f(m)): V \in \mathcal{U}_{Y}\right\}\right) \leq \bigwedge\left\{U(m): U \in \mathcal{U}_{X}\right\} \leq U(m)$. Since $\mathcal{U} \in \operatorname{SQUNIF}(\mathcal{C}, \mathcal{M}), V=c_{Y}^{\mathcal{U}} \in \mathcal{U}_{Y}$ and $f^{-1}(V(f(m))) \leq U(m)$.

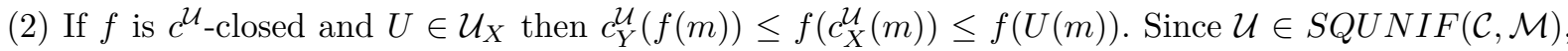
there is $V=c_{Y}^{\mathcal{U}} \in \mathcal{U}_{Y}$ such that $V(f(m)) \leq f(U(m))$. On the other hand if for any $U \in \mathcal{U}_{Y}$ there is $V \in \mathcal{U}_{Y}$ such that $V(f(m)) \leq f(U(m))$, then $c_{Y}^{\mathcal{U}}(f(m)) \leq f(U(m)) \Rightarrow c_{Y}^{\mathcal{U}}(f(m)) \leq f\left(\bigwedge\left\{U(m): U \in \mathcal{U}_{Y}\right\}\right)=$ $f\left(c_{X}^{\mathcal{U}}(m)\right)$.

One can show from Theorem 3.3 that $\operatorname{ICL}(\mathcal{C}, \mathcal{M})$ embeds as a sublattice in $Q U N I F(\mathcal{C}, \mathcal{M})$. First, we take a closer look at the lattice structure of $Q U N I F(\mathcal{C}, \mathcal{M})$, the conglomerate of all quasi-uniform structures on $\mathcal{C}$.

As noted in section two, $Q U N I F(\mathcal{C}, \mathcal{M})$ is ordered as follows: $\mathcal{U} \leq \mathcal{V}$ if for all $U \in \mathcal{U}_{X}$ there is $V \in \mathcal{V}_{X}$ such that $V(m) \leq U(m)$ for any $m \in \operatorname{subX}$. This order confers to $Q U N I F(\mathcal{C}, \mathcal{M})$ the structure of a complete lattice.

Theorem 3.5. Let $\mathcal{A}=\left\{\mathcal{U}^{i} \mid i \in I\right\} \subseteq Q U N I F(\mathcal{C}, \mathcal{M})$. Then $\mathcal{B}=\left\{\mathcal{B}_{X} \mid X \in \mathcal{C}\right\}$ with $\mathcal{B}_{X}=\left\{U_{1} \wedge \ldots \wedge\right.$ $U_{n} \mid$ for every $1 \leq i \leq n, U^{i} \in \mathcal{U}_{X}^{i}$ for some $\mathcal{U}^{i} \in \mathcal{A}$ and $\left.n \in \mathbb{N}\right\}$ is a base for the supremum $\mathcal{U}=\mathcal{A}$ of $\mathcal{A}$. If each $\mathcal{U}^{i}$ is a uniformity (resp transitive quasi-uniformity) on $\mathcal{C}$ then $\mathcal{U}$ is also a uniformity (resp. transitive quasi-uniformity).

Proof. (U1) and (U4) are clearly satisfied. For $(U 2)$, let $U=\wedge_{i}^{n} U^{i} \in \mathcal{B}_{X}^{i}, U^{i} \in \mathcal{U}^{i}$ for some $\mathcal{U}^{i} \in \mathcal{A}$. Then there are $V^{1}, \ldots, V^{n}$ such that $V^{i} \circ V^{i} \leq U^{i}$. Now, $V=\wedge_{i}^{n} V^{i}$ and $V \circ V \leq \wedge_{i}^{n}\left(V^{i} \circ V\right) \leq U$. Let $f: X \longrightarrow Y$ be a $\mathcal{C}$-morphism and $U=\wedge_{i}^{n} U^{i} \in \mathcal{B}_{Y}^{i}$. Then there are $V^{1}, \ldots, V^{n}$ such that $f\left(U^{i}(m)\right) \leq V^{i}(f(m))$. Thus $\left.f(U(m))=f\left(\wedge_{i}^{n} U^{i}(m)\right) \leq \wedge_{i}^{n} f\left(U^{i}(m)\right) \leq \wedge_{i}^{n} V^{i}(f(m)) \leq\left(\wedge_{i}^{n} V^{i}\right)(f(m))=V(f(m))\right)$. It is clear that $\mathcal{U}$ is finer than each $\mathcal{U}^{i}$ and if $\mathcal{V}$ is another quasi-uniformity on $\mathcal{C}$ that is finer than each $\mathcal{U}^{i}$, then $\mathcal{U}$ is coarser than $\mathcal{V}$.

Let each $\mathcal{V}^{i}$ be a uniformity and $p \leq U(m)$ for any $U \in \mathcal{B}_{X}$ with $p, m \in \operatorname{sub} X$. Then $p \leq \wedge_{i}^{n} U^{i}(m)$ for any $U^{i} \in \mathcal{U}^{i}$, for some $\mathcal{U}^{i} \in \mathcal{A}$ and $n \in \mathbb{N}$ and there are $V^{1}, \ldots, V^{n}$ with $m \leq U^{i}(p)$ for each $i$. Hence $m \leq \wedge_{i}^{n} V^{i}(p)=V(p)$. Assume that for each $i, \mathcal{U}^{i} \in T Q U N I F(\mathcal{C}, \mathcal{M})$ and $U \in \mathcal{U}_{X}$. Then $U=\wedge_{i}^{n} U^{i}$, $U^{i} \in \mathcal{U}_{X}^{i}$, for some $\mathcal{U}^{i} \in \mathcal{A}$. Since $U(U(m)) \leq U^{i}$ for each $i, U(U(m)) \leq \wedge_{i}^{n} U^{i}(m)=U(m)$.

Corollary 3.6. $Q U N I F(\mathcal{C}, \mathcal{M})$ is a complete lattice.

Proof. The least element is $\mathcal{I}_{X}=\mathbf{1}_{\mathbf{X}}$ for any $X \in \mathcal{M}$ while the greatest is the quasi-uniformity $\mathcal{D}_{X}=$ $\left\{U \in \mathcal{F}(\operatorname{subX}) \mid \mathbf{d}_{\mathbf{X}} \leq \mathbf{U}\right\}$. For any $\mathcal{A}=\left\{\mathcal{U}_{i} \mid i \in I\right\} \subseteq Q U N I F(\mathcal{C}, \mathcal{M}), \mathcal{U}=\bigvee \mathcal{A}$ of $\mathcal{A}$ is constructed as in Theorem 3.5. Thus $Q U N I F(\mathcal{C}, \mathcal{M})$ ) is a complete lattice since the meet can be constructed as the join of all upper bounds of $\mathcal{A}$.

A reader familiar with the theory of closure operators would have noticed that $\mathbf{d}_{\mathbf{X}}$ and $\mathbf{1}_{\mathbf{X}}$ in Corollary 3.6 , as defined earlier, are the discrete and trivial closure operators, respectively (see e.g. [4]).

Corollary 3.7. $\operatorname{UNIF}(\mathcal{C}, \mathcal{M}))$ and $\operatorname{TQUNIF}(\mathcal{C}, \mathcal{M}))$ are complete sublattices of $Q U N I F(\mathcal{C}, \mathcal{M}))$.

Theorem 3.8. The conglomerate $U N I F(\mathcal{C}, \mathcal{M}))$ and $T Q U N I F(\mathcal{C}, \mathcal{M}))$ are correflective in $Q U N I F(\mathcal{C}, \mathcal{M}))$.

Theorem 3.9. Let $\left.c, c^{\prime} \in \operatorname{ICL}(\mathcal{C}, \mathcal{M})\right)$. Then $\mathcal{U}^{c} \bigvee \mathcal{U}^{c^{\prime}}=\mathcal{U}^{c \wedge c^{\prime}}$ and $\mathcal{U}^{c} \wedge \mathcal{U}^{c^{\prime}}=\widehat{\mathcal{U}^{c \wedge c^{\prime}}}$ where $\widehat{c \wedge c^{\prime}}$ is the idempotent hall of $c \wedge c^{\prime}$. 


\section{The Császár-Pervin quasi-uniform structure}

Proposition 4.1. Let $\mathcal{U}$ be a quasi-uniformity on $\mathcal{C}$ and $X \in \mathcal{C}$. Then $\sqsubset_{X}^{\mathcal{U}}=\bigcup\left\{\sqsubset_{X}^{U}: U \in \mathcal{U}_{X}\right\}$ is an interpolative topogenous order. Consequently

(i) $c_{X}^{\mathcal{U}}(m)=\bigwedge\left\{U(m): U \in \mathcal{U}_{X}\right\}$ is an idempotent closure operator on $\mathcal{C}$.

(ii) If for any $\mathcal{C}$-morphism $f: X \longrightarrow Y, f^{-1}$ commutes with the joins of subobjects, then $i^{\mathcal{U}}(m)=\bigvee\{p \in$ subX $U(p) \leq m$ for some $\left.U \in \mathcal{U}_{X}\right\}$ is an idempotent interior operator on $\mathcal{C}$.

The above proposition motivates the next definition.

Definition 4.1. Let $\mathcal{U} \in Q U N I F(\mathcal{C}, \mathcal{M})$. We shall say that $\mathcal{U}$ is compatible with a topogenous order $\sqsubset$ or the topogenous order $\sqsubset$ is induced by $\mathcal{U}$ if $\sqsubset_{X}=\bigcup\left\{\sqsubset_{X}^{U}: U \in \mathcal{U}_{X}\right\}$ for all $X \in \mathcal{C}$.

Because of Proposition 2.1, we can say that $\mathcal{U} \in Q U N I F(\mathcal{C}, \mathcal{M})$ is compatible with a closure operator $c$ (interior $i$ ) if $c_{X}(m)=\bigwedge\left\{U(m): U \in \mathcal{U}_{X}\right\}\left(i_{X}(m)=\bigvee\left\{n \mid U(n) \leq m\right.\right.$ for some $\left.U \in \mathcal{U}_{X}\right\}$ ) for any $m \in \operatorname{subX}$. One observes from Theorem 3.3 that given an idempotent closure operator $c$ on $\mathcal{C}$ there is a quasi-uniformity with a unique base $\mathcal{B}$, where $\mathcal{B}_{X}=\left\{c_{X}\right\}$ for any $X \in \mathcal{C}$, which induces $c$. Since each member of a base for the Császár-Pervin quasi-uniformity of a topological space depends on a finite number of open sets, we wish to depart from an interior operator $i$ (closure $c$ ) and form a base $\mathcal{B}$ of a quasi-uniformity on $\mathcal{C}$ such that each member of $\mathcal{B}$ depends on a somehow finite number of $i$-open ( $c$-closed) subobjects. As our subobject lattices are not boolean algebras in general, we make use of the categorical notion of syntopogenous structure to avoid complements that are used in the classical case.

Let us start by recalling from [11] a concept that provides a common categorical treatment of the notions of $i$-open and $c$-closed. For $\sqsubset \in T O R D(\mathcal{C}, \mathcal{M})$, a subobject $m$ of an object $X \in \mathcal{C}$ is $\sqsubset$-strict if $m \sqsubset_{X} m$.

Lemma 4.2. Let $X \in \mathcal{C}$ and $\mathbf{A}^{\sqsubset}=\{m \in$ subX $\mid m$ is $\sqsubset-$ strict $\}$.

(1) If $\sqsubset \in \wedge-\operatorname{INTORD}(\mathcal{C}, \mathcal{M})$ or $\sqsubset \in \bigvee-I N T O R D(\mathcal{C}, \mathcal{M})$, then $\mathcal{A}^{\sqsubset}$ is complete sublattice of subX.

(2) If $\sqsubset \in \bigvee-I N T O R D(\mathcal{C}, \mathcal{M}), m$ is $\sqsubset$-strict if and only if $i_{\bar{X}}(m)=m$

(3) If $\sqsubset \in \bigwedge-I N T O R D(\mathcal{C}, \mathcal{M})$, then $m$ is $\sqsubset$-strict if and only if $c_{X}^{\sqsubset}(m)=m$.

Theorem 4.3. Let $\sqsubset \in \bigvee-I N T O R D(\mathcal{C}, \mathcal{M})$. Then there is a transitive quasi-uniformity $\mathcal{U}$ on $\mathcal{C}$ compatible with $\sqsubset$.

Proof. Let $\sqsubset \in \bigvee-I N T O R D(\mathcal{C}, \mathcal{M})$. Then for any $X \in \mathcal{C}, \mathcal{A}^{\sqsubset}$ is a complete sublattice of $\operatorname{subX}$. If $F\left(\mathcal{A}^{\sqsubset}\right)$ is the collection of all finite sublattices of $\mathcal{A}^{\sqsubset}$, then $\mathcal{S}_{X}=\left\{\sqsubset_{X}^{L} \mid L \in F\left(\mathcal{A}^{\complement}\right)\right\}$ where $m \sqsubset_{X}^{L} n \Leftrightarrow \exists p \in L \mid m \leq$ $p \leq n$ is an interpolative co-perfect syntopogenous structure. By Theorem 2.2, $\mathcal{B}_{X}^{S}=\left\{U^{\sqsubset} \mid L \in F\left(\mathcal{A}^{\sqsubset}\right)\right\}$ is a base for a transitive quasi-uniformity $\mathcal{U}^{S}$ on $\mathcal{C}$. Now, let $\mathbb{P}=\mathcal{U}^{\mathcal{S}}$ then $m \sqsubset_{X}^{\mathcal{U}} n \Leftrightarrow \exists L \in F\left(\mathcal{A}^{\sqsubset}\right) \mid U^{\sqsubset^{L}}(m) \leq$ $n \Leftrightarrow m \sqsubset_{X}^{L} n \Leftrightarrow \exists p \in L \mid m \leq p \sqsubset_{X} p \leq n$. On the other hand, $m \sqsubset_{X} n \Leftrightarrow m \leq i \check{\Sigma}_{X}(n) \Rightarrow m \sqsubset_{X}$ $i_{X}^{\sqsubset}(m) \sqsubset_{X} n$. Since $i_{X}^{\sqsubset}(m) \in \mathcal{A}^{\sqsubset}$, put $L=\left\{i_{X}^{\sqsubset}(m), 0_{X}\right\}$ to have that $m \sqsubset_{X}^{\mathcal{U}} n \Leftrightarrow U^{{ }^{L}}(m) \leq n \Leftrightarrow m \sqsubset_{X}^{U} n$. Thus $\sqsubset^{\mathbb{P}}=\sqsubset$.

In a similar way to the above, we prove the Theorem.

Theorem 4.4. Let $\sqsubset \in \wedge-I N T O R D(\mathcal{C}, \mathcal{M})$. Then there is a transitive quasi-uniformity $\mathcal{U}$ on $\mathcal{C}$ compatible with $\sqsubset$ 
Theorems 4.3 and 4.4 are very important. On the one hand Theorem 4.3 allows to conclude that for any $i \in \operatorname{IINT}(\mathcal{C}, \mathcal{M})$, there is a transitive quasi-uniformity $\mathcal{U}$ on $\mathcal{C}$ compatible with $i$ while Theorem 4.4 provides us with another transitive quasi-uniformity compatible $c \in \operatorname{ICL}(\mathcal{C}, \mathcal{M})$. On the other hand they present a categorical version of the well known Császár-Pervin $([2,13])$ quasi-uniformity and its inverse. In the light of our previous observation that a quasi-uniformity on $\mathcal{C}$ is collection of families of closure operators, Theorems 4.3 and 4.4 say that given an idempotent interior operator $i$ (closure $c$ ), one can construct a family of idempotent closure operators determined by $i$ (c). This understanding allows one to apply these results to categories outside topology. Furthermore, the analysis of the proof of Theorem 4.3 allows to obtain a categorical generalization of A. Császár theorem (see [3]), which characterizes a topogenous order that is compatible with a transitive quasi-uniformity.

Theorem 4.5. Let $\sqsubset \in \operatorname{TORDC}(\mathcal{C}, \mathcal{M})$. Then $\sqsubset$ is compatible with $\mathcal{U} \in T Q U N I F C(\mathcal{C}, \mathcal{M})$ if and only if $\sqsubset=\sqsubset^{A}$ for some complete sublattice $\mathcal{A}$ of subX, where $m \sqsubset_{X}^{\mathcal{A}} n \Leftrightarrow \exists p \in \mathcal{A} \mid m \leq p \leq n$.

Proof. Assume that $\mathcal{U} \in T Q U N I F(\mathcal{C}, \mathcal{M})$ and $\sqsubset=\sqsubset^{U}$. For any $X \in \mathcal{C}$, let $\mathcal{A}=\mathcal{A}^{\subset}$. If $m \in \sqsubset_{X} n$ then there is $U \in \mathcal{U}_{X}$ such that $U(m) \leq n$. By assumption, $U(m) \sqsubset U(m) \Rightarrow U(m) \in \mathcal{A}$ so that $m \sqsubset_{X}^{\mathcal{A}} n$. On the other hand, $m \sqsubset_{X}^{\mathcal{A}} n \Rightarrow \exists p \in \mathcal{A}$ such that $m \leq p \sqsubset_{X} p \leq n \Rightarrow m \sqsubset_{X} n$. Conversely let $\mathcal{A}$ be a complete sublattice of $\operatorname{sub} X$ for any $X \in \mathcal{C}$ and $\sqsubset=\sqsubset^{\mathcal{A}}$. Let $F(\mathcal{A})$ be the collection of all finite sublattices $L$ of $\mathcal{A}$. Then $S \in \bigwedge-I N T C S Y N T(\mathcal{C}, \mathcal{M})$ where $\mathcal{S}_{X}=\left\{\sqsubset_{X}^{L} \mid L \in \mathcal{F}(A)\right\}$ and $m \in \sqsubset_{X} n \Leftrightarrow \exists p \in L \mid m \leq p \leq n$. By Theorem 2.2, $\mathcal{U}_{X}^{\mathcal{A}}=\left\{U^{\sqsubset^{L}} \mid L \in F(\mathcal{A})\right\}$ is a base for a transitive quasi-uniformity on $\mathcal{C}$. If $m \sqsubset_{X} n$, then there exists $p \in \mathcal{A}$ such that $m \leq p \leq n$. Put $L=\left\{0_{X}, p, 1_{X}\right\}$ to have $m \sqsubset_{X}^{\mathcal{U}^{\mathcal{A}}} n$. On the other hand if $m \sqsubset_{X}^{L} n$ for some $L \in F(\mathcal{A})$, then there is $p \in L$ such that $m \leq p \leq n \Rightarrow m \sqsubset_{X} n$ since $\sqsubset^{\mathcal{U}}=\bigcup\left\{\sqsubset^{U}: U \in \mathcal{U}_{X}\right\}$. Thus $\sqsubset=\sqsubset^{\mathcal{U}^{\mathcal{A}}}$.

From Theorems 4.3 and 4.4 and Proposition 2.1, we can characterize a closure operator (interior) compatible with a transitive quasi-uniformity.

Corollary 4.6. Let $i \in I N T(\mathcal{C}, \mathcal{M})$. Then $i$ is compatible with $\mathcal{U} \in \operatorname{TQUNIF}(\mathcal{C}, \mathcal{M})$ if and only if for any $X \in \mathcal{C}$ and $m \in \operatorname{subX}, i_{X}(m)=\bigvee\{n \mid \exists p \in \mathcal{A}: n \leq p \leq m\}$ for some complete sublattice $\mathcal{A}$ of subX.

Corollary 4.7. Let $c \in C L(\mathcal{C}, \mathcal{M})$. Then $c$ is compatible with $\mathcal{U} \in T Q U N I F(\mathcal{C}, \mathcal{M})$ if and only if for any $X \in \mathcal{C}$ and $m \in \operatorname{subX}, c_{X}=\bigwedge\{n \mid \exists p \in \mathcal{A}: m \leq p \leq n\}$ for some complete sublattice $\mathcal{A}$ of subX.

Proposition 4.8. Let $\sqsubset \in \operatorname{TORD}(\mathcal{C}, \mathcal{M})$. There is $\mathcal{U} \in Q U N I F(\mathcal{C}, \mathcal{M})$ such that $\mathcal{U}$ is compatible with $\sqsubset$ if and only if $\mathcal{A} \sqsubset$ is a complete sublattice of subX for any $X \in \mathcal{C}$.

Corollary 4.9. Let $c \in C L(\mathcal{C}, \mathcal{M})$. Then $c \in I C L(\mathcal{C}, \mathcal{M})$ if and only if for any $X \in \mathcal{C}$ there is a complete sublattice $\mathcal{A}$ of subX such that $\sqsubset_{X}^{c}=\sqsubset_{X}^{\mathcal{A}}$.

\section{Examples}

(1) Consider $\mathcal{C}=$ Top the category of topological spaces and continuous maps with $\mathcal{M}$ the class of embeddings and (of course) $\varepsilon$ the class of surjective continuous maps. For $A, B \subseteq X$ :

(a) Let $\sqsubset=\left\{\sqsubset_{X} \mid X \in\right.$ Top $\}$ with $A \sqsubset_{X} B \mid A \subseteq O \subseteq B$ for some $O \subseteq \mathcal{T}_{X}$. Then $\sqsubset \in$ $\bigvee-I N T O R D(\operatorname{Top}, \mathcal{M})$ and $F\left(\mathcal{A}^{\sqsubset}\right)=$ collection of all finite sublattices of $O X$ where $O X$ is collection of open subsets of $X$. Now $\mathcal{S}_{X}=\left\{\sqsubset_{X}^{L} \mid L \in F\left(\mathcal{A}^{\sqsubset}\right)\right\}$ with $A \sqsubset_{X}^{L} B \Leftrightarrow \exists O \in L \mid A \subseteq O \subseteq B$ is an interpolative co-perfect syntopogenous structure. By Theorem 2.2, $\mathcal{B}_{X}^{S}=\left\{U^{\sqsubset^{L}} \mid L \in F\left(\mathcal{A}^{\sqsubset}\right)\right\}$ is a base for a transitive quasi-uniformity $\mathcal{U}$ on Top. One uses the observation that 


$$
y \in U^{\sqsubset^{L}}[x] \Longleftrightarrow\{x\} \sqsubset_{X}^{L} X \backslash\{y\}
$$

is not true to see that $\mathcal{B}_{X}^{S}$ is a base for the Pervin quasi-uniformity $\mathcal{P}$ of $\mathcal{T}_{X}$.

(b) Let $\sqsubset=\left\{\sqsubset_{X} \mid X \in \mathbf{T o p}\right\}$ with $A \sqsubset_{X} B \Leftrightarrow A \subseteq C \subseteq B$ for some $C \subseteq \mathcal{T}_{X}$. Then $\bigwedge-I N T O R D(\operatorname{Top}, \mathcal{M})$. Since $\mathcal{A}^{\sqsubset}=\left\{C \subseteq X \mid C\right.$ is closed in $\left.\mathcal{T}_{X}\right\}$ is a complete lattice, $\mathcal{S}_{X}=$ $\left\{\sqsubset_{X}^{L} \mid L \in F\left(\mathcal{A}^{\sqsubset}\right)\right\}$ with $A \sqsubset_{X}^{L} B \Leftrightarrow \exists C \in L \mid A \subseteq C \subseteq B$ is an interpolative co-perfect syntopogenous structure and so $\mathcal{B}_{X}^{S}=\left\{U^{\sqsubset^{L}} \mid L \in F\left(\mathcal{A}^{\sqsubset}\right)\right\}$ where $(x, y) \notin U^{\sqsubset^{L}} \Leftrightarrow\{x\} \sqsubset_{X}^{L} X \backslash\{y\}$ is a base for a transitive quasi-uniformity $\mathcal{U}$ on Top. Since $\mathcal{P}^{-1}$ is generated by $\left\{\mathcal{S}_{C} \mid C\right.$ is closed in $\left.\mathcal{T}\right\}$ and $\mathcal{S}_{C}=((X \backslash C) \times X) \cup X \times C, \mathcal{U}=\mathcal{P}^{-1}$.

(2) Let $\mathcal{C}=$ Grp be the category of groups and group homomorphisms with (surjective, injective)factorisation system. For any $A, B \leq G$, let $\sqsubset_{G}=\left\{\sqsubset_{G} \mid G \in \mathbf{G r p}\right\}$ with $A \sqsubset_{G} B \Leftrightarrow A \leq N \leq B$ with $N \triangleleft G$ is a meet preserving topogenous order on Grp. Then $A^{\sqsubset}=\{N \leq G \mid N \triangleleft G\}$ is a complete lattice so that $\mathcal{S}_{X}=\left\{\sqsubset_{X}^{L} \mid L \in F\left(\mathcal{A}^{\sqsubset}\right)\right\}$ where $A \sqsubset_{X}^{L} B \Leftrightarrow \exists N \in L \mid A \subseteq N \subseteq B$ is an interpolative co-perfect syntopogenous structure, that is each $U^{\sqsubset^{L}}(A)=\cap\{B \leq G \mid \exists N \in L: A \leq N \leq B\}$ is an idempotent closure operator on Grp. Moreover, $\bigcap\left\{U^{\sqsubset^{L}}(A) \mid L \in F\left(\mathcal{A}^{\sqsubset}\right)\right\}=\mathcal{N}_{G}(\bar{A})$ the normal closure.

(3) Let $\mathcal{C}=$ Ring be the category of rings and their homomorphisms with (surjective, injective)-factorisation system. For any $B, C \leq A$, let $\sqsubset=\left\{\sqsubset_{A} \mid A \in \mathbf{R i n g}\right\}$ with $B \sqsubset_{A} B \Leftrightarrow B \leq I \leq B$ with $I$ an ideal of $A$. $\sqsubset_{A}$ is clearly a meet preserving topogenous order on Ring. Now $F\left(A^{\sqsubset}\right)=\{I \leq A \mid I$ is an ideal of $A\}$ is a complete lattice so that $\mathcal{S}_{X}=\left\{\sqsubset_{X}^{L} \mid L \in F\left(\mathcal{A}^{\sqsubset}\right)\right\}$ where $B \sqsubset_{X}^{L} C \Leftrightarrow \exists I \in L \mid B \subseteq I \subseteq C$ is an interpolative co-perfect syntopogenous structure and each $U^{\sqsubset^{L}}(A)=\cap\{B \leq G \mid \exists I \in L: B \leq I \leq C\}$ is an idempotent closure operator on Ring.

\section{References}

[1] J. Adámek, H. Herrlich, G.E. Strecker, Abstract and Concrete Categories: The Joy of Cats, Repr. Theory Appl. Categ., vol. 17, 2006, pp. 1-507, Reprint of the 1990 original [Wiley, New York].

[2] Á. Császár, Foundations of General Topology, A Pergamon Press Book. The Macmillan Co., New York, 1963.

[3] Á. Császár, Transitive quasi-uniformities and topogenous orders, Acta Math. Hung. 86 (1-2) (2000) 83-87.

[4] D. Dikrajan, W. Tholen, Categorical Structure of Closure Operators with Applications to Topology, Algebra and Discrete Mathematics, Mathematics and Its Applications., vol. 346, Kluwer Academic Publishers Group, Dordrecht, 1995.

[5] D. Dikranjan, E. Giuli, Closure operators I, Topol. Appl. 27 (2) (1987) 129-143.

[6] C. Dowker, Mappings of proximity structures, in: General Topology and Its Relations to Modern Analysis and Algebra, 1962, pp. 139-141.

[7] P. Fletcher, W.F. Lindgren, Quasi-Uniform Spaces, Lecture Notes in Pure Appl. Math., vol. 77, Dekker, New York, 1982.

[8] D. Holgate, M. Iragi, Quasi-uniform and syntopogenous structures on categories, Topol. Appl. 263 (2019) 16-25.

[9] D. Holgate, M. Iragi, A. Razafindrakoto, Topogenous and nearness structures on categories, Appl. Categ. Struct. 24 (2016) 447-455.

[10] D. Holgate, J. Šlapal, Categorical neighborhood operators, Topol. Appl. 158 (17) (2011) 2356-2365.

[11] M. Iragi, Topogenous structures on categories, MSc Thesis, University of the Western Cape, 2016.

[12] M. Iragi, Quasi-uniform and syntopogenous structures on categories, PhD Thesis, University of the Western Cape, 2019.

[13] W.J. Pervin, Quasi-uniformization of topological spaces, Math. Ann. 147 (1962) 316-317.

[14] S.J.R. Vorster, Interior operators in general categories, Quaest. Math. 23 (4) (2000) 405-416. 